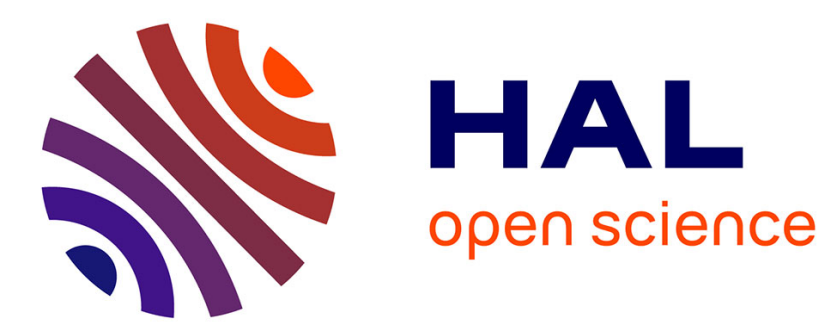

\title{
Algorithms for cable-suspended payload sway damping by vertical motion of the pivot base
}

Matěj Kuře, Jaroslav Bušek, Tomas Vyhlidal, Silviu-Iulian Niculescu

\section{To cite this version:}

Matěj Kuře, Jaroslav Bušek, Tomas Vyhlidal, Silviu-Iulian Niculescu. Algorithms for cable-suspended payload sway damping by vertical motion of the pivot base. Mechanical Systems and Signal Processing, 2021, 149, pp.107131. 10.1016/j.ymssp.2020.107131 . hal-03108536

\section{HAL Id: hal-03108536 \\ https://hal-centralesupelec.archives-ouvertes.fr/hal-03108536}

Submitted on 6 May 2021

HAL is a multi-disciplinary open access archive for the deposit and dissemination of scientific research documents, whether they are published or not. The documents may come from teaching and research institutions in France or abroad, or from public or private research centers.
L'archive ouverte pluridisciplinaire HAL, est destinée au dépôt et à la diffusion de documents scientifiques de niveau recherche, publiés ou non, émanant des établissements d'enseignement et de recherche français ou étrangers, des laboratoires publics ou privés. 


\title{
Algorithms for Cable-Suspended Payload Sway Damping by Vertical Motion of the Pivot Based
}

\author{
Matěj Kuře ${ }^{a, b}$ Jaroslav Bušek $^{\mathrm{a}, \mathrm{b}}$ Tomáš Vyhlídal ${ }^{\mathrm{a}, \mathrm{b}}$ \\ Silviu-Iulian Niculescu ${ }^{\mathrm{c}}$ \\ ${ }^{a}$ Dept. of Instrumentation and Control Eng., Faculty of Mechanical Engineering, \\ Czech Technical University in Prague, Technická 1902/4, 166 07 Praha 6, Czech \\ Republic, (Matej.Kure, Jaroslav.Busek, Tomas.Vyhlidal)@fs.cvut.cz \\ ${ }^{\mathrm{b}}$ Czech Institute of Informatics, Robotics and Cybernetics, Czech Technical \\ University in Prague, Jugoslávských partyzánů 1580/3, 16000 Praha 6, Czech \\ Republic, \\ ${ }^{\mathrm{c}}$ Université Paris-Saclay, CNRS-CentraleSupélec, Laboratoire des Signaux et \\ Systèmes, 91192 Gif-sur-Yvette, France. The author is also a member of the Inria \\ team "DISCO (Inria-CNRS-CentraleSupélec) \\ Silviu.Niculescu@12s.centralesupelec.fr
}

\begin{abstract}
The solution of a case study problem of suspended payload sway damping by moving a pivot base in vertical direction is presented. Unlike for the classical problem of anti-sway control for moving the base in the horizontal direction, implemented e.g. in cranes, a direct solution by using control feedback theory for linear systems is not possible. Once the model is linearized, it becomes uncontrollable. Thus, a derivation of a nonlinear controller is needed to solve the task. In this context, two solutions are proposed. The first solution is based on imposing harmonic motion of the base with double frequency of the payload natural frequency. Synchronization of the base and the payload deflection angle is done either by proportional time-delay controller or by proportional-derivative delay free controller. Secondly, the Lyapunov's second method is directly applied to derive a nonlinear controller. For both cases, balancing the dissipated energy, rules for determining equivalent damping are explicitly derived. After discussing and solving the corresponding implementation aspects, both simulation and experimental validations are performed. The experimental validation is performed on a simplified problem, where only horizontal motion is possible. The simulation based validation is performed on a nonlinear two dimensional model of a quadcopter carrying a suspended payload.
\end{abstract}

Paper accepted for publication in Mechanical Systems and Signal Processing, Elsevier, 2021. 
Key words: anti-sway control system, suspended load, nonlinear control, time delay, Lyapunov method, mechatronic system

\section{Introduction}

Suspended payload sway damping task has particularly been associated with crane applications [1]. The pre-compensation of payload oscillation during horizontal maneuvers of the crane trolley can be effectively solved by input shaping, see, for instance, [2], [3], [4]. In particular among other contributions, in [5], input shaping is applied to payload sway compensation when carried by a helicopter. In input shaping, the trajectory of the base (trolley) is shaped by a time delay filter so that the payload does not sway as soon as the trolley reaches the target position. As the input shaping is a feed-forward technique, it is not effective when the payload sway is excited by external disturbances. This task can be solved by combined input shaping and feedback control [6], [7], or embedding the inverse shaper to the control feedback [8]. Direct feedback control methods to avoid payload sway during maneuvers have been proposed e.g. in [9] with real-time visual tracking, in [10] by nonlinear tracking control and in [11] by MPC constrained algorithm. A backstepping method was proposed in [12] to derive a robust controller for trajectory tracking for a quadcopter with suspended load. The more advanced control task with variable cable length has been addressed in [13] by sliding mode approach, in [14] by partial feedback linearization, and in [15], [16] by using appropriate Lyapunov-based methods. In these works, the cable length is adjusted simultaneously with the trolley position to obtain improved transient characteristics. A hybrid control design method was applied in [17] to perform lift maneuver for a quadcopter with a cable-suspended load.

In this work, a limit case at which the payload sway damping is achieved by vertical motion of the pivot base is addressed. Analogous tasks have been studied for pendulum-like applications, e.g. modeling of child or gymnast swing [18], [19]. In these applications, however, the base is considered fixed and pendulum motion control is performed by varying the pendulum effective length. An inverse problem of varying length pendulum to achieve periodic motion of the pendulum was studied in [20], [21]. The problem of pendulum damping by adjusting the cable length was studied by [22]. The corresponding method is based on a feed-forward control law generating periodic adjustment of the cable length. The experimental study of this concept was performed in [23] and [24]. It was shown theoretically and experimentally that, due to slight period variation of the physical pendulum, it is difficult to keep the synchronization of the cable length and the payload motion. This difficulty, which disqualifies the concept from the practical use, was removed in [25] where the 

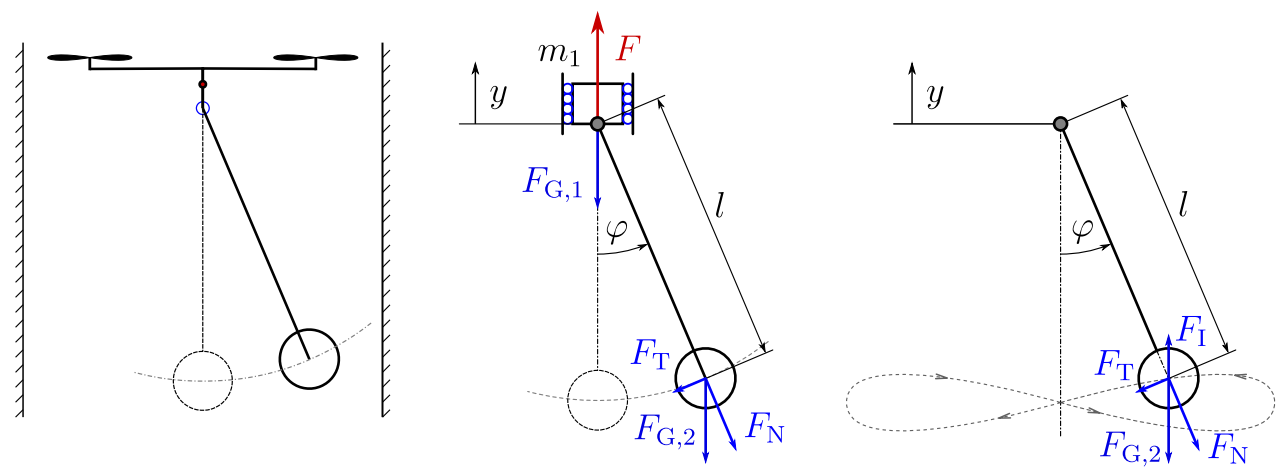

Fig. 1. Problem motivation and formulation: Left - Scheme of the constrained space for a quadcopter with suspended load. Mid - Simplified model to a pendulum with moving pivot base, Right - Further simplified model to a pendulum with a mass free moving pivot, with a desired trajectory of the pendulum bob to achieve sway damping.

feed-forward rule was turned to the construction of some appropriate nonlinear feedback solution. The robustness in sway damping of the proposed solution was thoroughly studied, including experimental validation. An alternative control design by Lyapunov methods was presented in [26], see also a recent study [27] and references therein. Note that by the Lyapunov approach, the amplitude decay does not achieve exponential character, as it is the case for the former method.

\subsection{Problem motivation and formulation}

Solving the pendulum sway damping by vertical motion of the pivot base is motivated by design of advanced control of an Unmanned Aerial Vehicles (UAVs) with a suspended payload. In [28] an algorithm was proposed to pre-compensate the payload oscillations by an input shaper in the horizontal motion of the quadcopter. However, it may happen that UAV gets into a situation, where the space for maneuvers is restricted, as shown in Fig. 1-Left, for example in narrow corridors, forest or moving in some uncertain environments. Then, the sway damping is possible either by adjusting the cable length or by vertical motion of the base. The former problem was thoroughly addressed as outlined above. The latter, which is more suitable for UAVs, is solved in this paper.

To develop a control concept for damping the UAV suspended payload sway by vertical motion, we simplify the set-up (as shown in Fig. 1-Left) into a model of a pendulum with vertically moving pivot base shown in Fig. 1Mid. The vertically moving pivot has mass $m_{1}$ with attached mathematical pendulum of length $l$. There is a bob of mass $m_{2}$ at the end of pendulum. The angle between the pendulum and vertical axis of the inertial coordinate 
system is denoted as $\varphi$. The control action is performed by the force $F$ that also keeps the system from free falling by pre-compensating the gravity force.

The corresponding non-linear model of the system writes as follows

$$
\begin{gathered}
\ddot{\varphi}+\frac{g}{l} \sin \varphi+\frac{\ddot{y}}{l} \sin \varphi=0, \\
\left(m_{1}+m_{2}\right) \ddot{y}+b \dot{y}=F-\left(m_{1}+m_{2}\right) g-m_{2} l\left(\ddot{\varphi} \sin \varphi+\dot{\varphi}^{2} \cos \varphi\right),
\end{gathered}
$$

where $b$ represents the viscous damping coefficient.

At a first step, for derivation of the reference for pivot motion, we assume that the pivot is of zero mass, as shown in Fig. 1-Right. For the theoretical design, only the first equation (1) is considered with $\ddot{y}$ being the system control input and $\varphi$ the output. In the validation part of the paper, the problem shown in Fig. 1-Mid and described by (1)-(2) is addressed by simulations and experiments. Finally, the simulation based validation is performed for a quadcopter with the suspended load, according to Fig. 1-Left.

The paper is organized as follows. Section 2 addresses some preliminary results concerning the problem of oscillation damping by adjusting the cable length. The main results are presented in Section 3 and Section 4, where two classes of algorithms are proposed and studied for damping the pendulum sway by motion of the pivot base. Next, Section 5 then provides case study validation. The paper is concluded by some brief summary in Section 6. It is worth mentioning that the problem motivation and a part of the theoretical results were presented in a conference paper [29]. As these results are experimentally validated in the Case study section 5 , we present them in Section 3 due to the consistency reasons.

\section{Preliminaries - Pendulum oscillation damping by adjusting the cable length}

The first method derived in the sequel is an adaptation of the method proposed in [22] for damping the pendulum bob oscillation by adjusting the cable length while considering fixed position of the pivot $(y=0)$. Neglecting the mass of the cable, friction in the pivot and the viscous friction corresponding to the pendulum motion, by balancing momentum, the governing model is given by the following nonlinear second-order equation

$$
\ddot{\varphi}+\frac{2 \dot{l} \dot{\varphi}}{l}+\frac{g}{l} \sin \varphi=0,
$$

where $\varphi, l$ denote the pendulum angle and the length of the cable, respectively. Stilling and Szyszkowski proposed in [22] to utilize the Coriolis force $F_{c}=$ 
$2 m i \dot{\varphi}$ to damp the oscillation leading to a harmonic motion of the bob shown in Fig. 1 - Right and given by

$$
l=l_{0}-\Delta l \sin (2 \Omega t),
$$

where $l_{0}$ is the nominal position, $\Delta l$ is the amplitude of the cable length adjustment and $\Omega=\sqrt{\frac{g}{l}}$ is the natural frequency of the linearized pendulum model. Coupling (3)-(4), they have also shown that, for small amplitudes of $\varphi$, the coupled system is of the dynamics corresponding to the damped secondorder oscillator

$$
\ddot{\varphi}+2 \zeta \Omega \dot{\varphi}+\Omega^{2} \varphi=0,
$$

where $\zeta$ is the damping with the value given in Lemma 1.

Lemma 1 ([22]) The equivalent damping of the system (3)-(4) is given by

$$
\zeta=\frac{3 \Delta l}{4 l}
$$

The above rule (6) results from balancing the dissipated energy of the ideal oscillator (5) and the coupled system (3)-(4) over one oscillation period.

\section{Damping pendulum oscillation by harmonic motion of the pivot}

For damping the pendulum oscillation by a motion of the pivot, we adopt the approach proposed in [22]. The pivot motion is considered in the form

$$
y=y_{0}+\Delta y \sin (2 \Omega t),
$$

where $y_{0}$ is the nominal position of the pivot and $\Delta y$ is the oscillation amplitude. The damping effect is achieved due to momentum impact of the inertia force in the direction $y$ when the bob trajectory shown in Fig. 1-Right imposed by (7) is followed. The control design objective is to select the value of $\Delta y$ so that the pendulum angle of the coupled system (1) and (7) is damped equivalently to (5) with a predefined damping $\zeta$.

Lemma 2 The equivalent damping of the system (1) and (7) is given by

$$
\zeta=\frac{\Delta y}{l}
$$

PROOF. Under small angle assumption, the model (1) can be simplified to

$$
\ddot{\varphi}+\Omega^{2} \varphi+\frac{1}{l} \ddot{y} \varphi=0 .
$$


Then, after multiplying equation (9) by $\dot{\varphi}$ and considering

$$
\dot{\varphi} \ddot{\varphi}=\frac{\mathrm{d}}{\mathrm{d} t}\left(\frac{1}{2} \dot{\varphi}^{2}\right)
$$

and

$$
\varphi \dot{\varphi}=\frac{\mathrm{d}}{\mathrm{d} t}\left(\frac{1}{2} \varphi^{2}\right)
$$

we turn the equation (9) to the form

$$
\frac{\mathrm{d}}{\mathrm{d} t}\left(\frac{1}{2} \dot{\varphi}^{2}\right)+\Omega^{2} \frac{\mathrm{d}}{\mathrm{d} t}\left(\frac{1}{2} \varphi^{2}\right)+\frac{1}{l} \ddot{y} \frac{\mathrm{d}}{\mathrm{d} t}\left(\frac{1}{2} \varphi^{2}\right)=0 .
$$

Integrating (12) over the oscillation period $t \in[0, T], T=\frac{2 \pi}{\Omega}$, we get

$$
\frac{1}{2} \dot{\varphi}^{2}(T)-\frac{1}{2} \dot{\varphi}^{2}(0)+\frac{1}{2} \Omega^{2} \varphi^{2}(T)-\frac{1}{2} \Omega^{2} \varphi^{2}(0)+\frac{1}{2 l} \int_{0}^{T} \ddot{y} \frac{\mathrm{d}}{\mathrm{d} t}\left(\varphi^{2}\right) \mathrm{d} t=0
$$

leading to

$$
-\frac{1}{2 l} \int_{0}^{T} \ddot{y} \frac{\mathrm{d}}{\mathrm{d} t}\left(\varphi^{2}\right) \mathrm{d} t=\frac{1}{2} \dot{\varphi}^{2}(T)+\frac{1}{2} \Omega^{2} \varphi^{2}(T)-\frac{1}{2} \dot{\varphi}^{2}(0)-\frac{1}{2} \Omega^{2} \varphi^{2}(0),
$$

representing the energy dissipation over the period $T$

$$
\Delta E=E(T)-E(0),
$$

where

$$
\Delta E=-\frac{1}{2 l} \int_{0}^{T} \ddot{y} \frac{\mathrm{d}}{\mathrm{d} t}\left(\varphi^{2}\right) \mathrm{d} t=-\frac{1}{2 l}\left[\ddot{y} \varphi^{2}\right]_{0}^{T}+\frac{1}{2 l} \int_{0}^{T} \dddot{y} \varphi^{2} \mathrm{~d} t
$$

Considering small damping and assuming $\dot{\varphi}(0)=0$, the corresponding motion of the oscillator (5) can be approximated by

$$
\varphi \cong \varphi_{0} \cos (\Omega t)
$$

where $\varphi_{0}=\varphi(0) \neq 0$ is the initial pendulum angle. According to the proposed damping rule (7), we get

$$
\begin{aligned}
& \dddot{y} \cong-4 \Delta y \Omega^{2} \sin (2 \Omega t), \\
& \dddot{y} \cong-8 \Delta y \Omega^{3} \cos (2 \Omega t) .
\end{aligned}
$$

Substituting (18)-(19) into (16) and assuming (17) we obtain

$$
\Delta E=-\frac{1}{2 l} 8 \Omega^{3} \Delta y \varphi_{0}^{2} \int_{0}^{T} \cos (2 \Omega t) \cos ^{2}(\Omega t) \mathrm{d} t=-\frac{1}{2 l} 8 \Omega^{3} \Delta y \varphi_{0}^{2} \frac{T}{4},
$$


which can be further simplified to the final equation for the energy dissipation over a single period

$$
\Delta E=-\frac{\Delta y}{l} 2 \pi \Omega^{2} \varphi_{0}^{2}
$$

In [22], it was derived that the ratio between dissipated energy $\Delta E$ over a single period and initial energy $E(0)$ of the oscillator (5) is given by

$$
\frac{\Delta E}{E(0)}=-4 \pi \zeta
$$

Considering the initial conditions $\varphi(0)=\varphi_{0}, \dot{\varphi}(0)=0$ and $\ddot{\varphi}(0)=0$, the initial energy in (14) is given by

$$
E(0)=\frac{1}{2} \Omega^{2} \varphi_{0}^{2}
$$

Substituting (21) and (23) into (22), we get

$$
\frac{\Delta E}{E(0)}=\frac{-\frac{\Delta y}{l} 2 \pi \Omega^{2} \varphi_{0}^{2}}{\frac{1}{2} \Omega^{2} \varphi_{0}^{2}}=-4 \pi \frac{\Delta y}{l} .
$$

Comparing (22) and (24) we obtain (8), which concludes the proof.

\subsection{Control feedback solution}

From the implementation point of view, the feedforward character of (7) cannot be used in practice. The main problem concerns the fact that the oscillation frequency is not constant for the nonlinear pendulum (1), but depends on the oscillation amplitude. Assuming $\ddot{y}=0$, the oscillation frequency $\bar{\Omega}$ of (1) with the period given by $\bar{T}=4 \sqrt{\frac{l}{2 g}} \int_{0}^{\varphi_{0}} \frac{1}{\sqrt{\cos \varphi-\cos \varphi_{0}}} \mathrm{~d} \varphi$, is slightly lower compared to the design frequency $\Omega$ of the ideal oscillator (5) with $\zeta=0$ and period $T=2 \pi \sqrt{\frac{l}{g}}$, [30]. Besides, in real applications, the oscillation period can also be affected by external disturbances, e.g. by wind. Therefore, adopting the method proposed in [25], the algorithm (7) is turned to the feedback form as explained in the sequel.

Considering the damping $\zeta$ is small, but nonzero, the response of the second-order oscillator (5) can be approximated by

$$
\varphi(t) \approx \varphi_{0} e^{-\zeta \Omega t} \cos (\Omega t) .
$$

From (25), the cosine function can be expressed by

$$
\cos (\Omega t) \approx \frac{e^{\zeta \Omega t}}{\varphi_{0}} \varphi(t)
$$


As a starting point, some standard trigonometric identities are applied

$$
\begin{aligned}
\sin (2 \Omega t) & =\cos \left(2\left(\Omega t-\frac{\pi}{4}\right)\right)=\cos ^{2}\left(\Omega t-\frac{\pi}{4}\right)-\sin ^{2}\left(\Omega t-\frac{\pi}{4}\right) \\
& =\cos ^{2}\left(\Omega t-\frac{\pi}{4}\right)-\cos ^{2}\left(\Omega t-\frac{\pi}{4}-\frac{\pi}{2}\right),
\end{aligned}
$$

which can be extended to

$$
\sin (2 \Omega t)=\frac{\cos ^{2}\left(\Omega t-\frac{\pi}{4}\right)-\cos ^{2}\left(\Omega t-\frac{\pi}{4}-\frac{\pi}{2}\right)}{\cos ^{2}\left(\Omega t-\frac{\pi}{4}\right)+\cos ^{2}\left(\Omega t-\frac{\pi}{4}-\frac{\pi}{2}\right)} .
$$

Substituting (26) to (28), the feedforward rule (7) can be turned to the feedback rule

$$
y(t)=y_{0}+\Delta y \frac{\varphi^{2}\left(t-\tau_{2}\right)-e^{-\zeta \pi} \varphi^{2}\left(t-\tau_{2}-\tau_{1}\right)}{\varphi^{2}\left(t-\tau_{2}\right)+e^{-\zeta \pi} \varphi^{2}\left(t-\tau_{2}-\tau_{1}\right)}
$$

where $\tau_{1}=\frac{\pi}{2 \Omega}$ and $\tau_{2}=\frac{\pi}{4 \Omega}$. Note that the extended form (28) is applied to cancel out the dependency on the initial angle $\varphi_{0}$ and the exponential term $e^{-\zeta \Omega t}$.

By analogy, a delay free feedback solution can be obtained. Applying the trigonometric identities

$$
\sin (2 \Omega t)=\frac{2 \cos (\Omega t) \sin (\Omega t)}{\cos ^{2}(\Omega t)+\sin ^{2}(\Omega t)}
$$

and differentiating (26), we obtain the approximation

$$
\sin (\Omega t) \cong-\frac{e^{\zeta \Omega t}}{\varphi_{0}}\left(\zeta \varphi(t)+\frac{1}{\Omega} \dot{\varphi}(t)\right)
$$

Substituting (26) and (31) into (30), the delay free feedback solution is given as

$$
y(t)=y_{0}-\Delta y \frac{2 \varphi(t)\left(\zeta \varphi(t)+\frac{1}{\Omega} \dot{\varphi}(t)\right)}{\varphi^{2}(t)+\left(\zeta \varphi(t)+\frac{1}{\Omega} \dot{\varphi}(t)\right)^{2}} .
$$

It is important to point out that for using delay-free rule, additional sensor that measures angular rate has to be considered. Alternatively a differentiation of the measured angle can be applied to approximate the derivative, however with the remark that it is an operation sensitive to measured noise. 


\section{Nonlinear control feedback design by Lyapunov approach}

As an alternative to the above rules based on approximation of the harmonic signal, a nonlinear controller directly based on the Lyapunov method is designed in this section. In the first step, the system (1) is turned to the state space formulation

$$
\begin{aligned}
& \dot{x}_{1}=x_{2} \\
& \dot{x}_{2}=-\Omega^{2} \sin x_{1}-\frac{1}{l} \ddot{y} \sin x_{1},
\end{aligned}
$$

where $x_{1}=\varphi$ and $x_{2}=\dot{\varphi}$ and $\ddot{y}$ is considered as the control input. A stabilizing feedback is proposed in the following Lemma.

Lemma 3 The nonlinear feedback control rule

$$
\ddot{y}=K \frac{x_{1} x_{2}}{x_{1}^{2}+\frac{1}{\Omega^{2}} x_{2}^{2}}
$$

with a parameter $K>0$ stabilizes locally the system (33) at the equilibrium point $x_{\mathrm{s}}=[0,0]^{T}$, assuming the given initial conditions $x_{1}(0) \in\left[-\frac{\pi}{2} ; \frac{\pi}{2}\right]$, $x_{2}(0)=0$.

PROOF. In order to prove that the feedback rule (34) stabilizes the system (33) in the bounded neighbourhood of the equilibrium point $x_{\mathrm{s}}=[0,0]^{T}$, we apply Lyapunov's second method. Define the Lyapunov candidate $V: \mathbb{R}^{2} \mapsto$ $\mathbb{R}_{+}$

$$
V(x)=2 \Omega^{2}\left(1-\cos x_{1}\right)+x_{2}^{2},
$$

satisfying $V\left(x_{\mathrm{s}}\right)=0$ and $V(x)>0$ for $x \neq 0$ assuming $x_{1} \in\left[-\frac{\pi}{2} ; \frac{\pi}{2}\right]$. Next, by differentiating (35), we obtain

$$
\dot{V}(x)=\frac{\partial V(x)}{\partial x_{1}} \dot{x}_{1}+\frac{\partial V(x)}{\partial x_{2}} \dot{x}_{2}=2 \Omega^{2} \sin x_{1} x_{2}+2 x_{2}\left(-\Omega^{2} \sin x_{1}-\frac{1}{l} \ddot{y} \sin x_{1}\right) .
$$

Simplifying the expression and substituting (34) for $\ddot{y}$,

$$
\dot{V}(x)=-\frac{2 K x_{2}^{2}}{l\left(x_{1}^{2}+\frac{1}{\Omega^{2}} x_{2}^{2}\right)} x_{1} \sin x_{1} .
$$

As $\dot{V} \leq 0$ for $K>0$ and $x_{1} \in\left[-\frac{\pi}{2} ; \frac{\pi}{2}\right]$, the stability on the neighborhood of the given equilibrium point $x_{\mathrm{s}}$ follows from the Lyapunov's second method [31]. Note that the equality $\dot{V}=0$ takes place either when passing the point $x_{1}=0$ at which $x_{2} \neq 0$ (indicating nonzero velocity), or at the turning points 
when $x_{2}=0$. It is also easy to derive by the L'Hopital's rule applied to (37) that $\dot{V}=0$ when reaching the equilibrium point $[0,0]^{T}$ at the limit case. The considered initial conditions $x_{1}(0) \in\left[-\frac{\pi}{2} ; \frac{\pi}{2}\right], x_{2}(0)=0$ imply that system motion stays in the neighbourhood of the considered equilibrium point, which is necessary assumption of the stability proof.

Remark 1 Let us note that when the load is cable-suspended, the assumption $x_{1} \in\left[-\frac{\pi}{2} ; \frac{\pi}{2}\right]$ covers fully all the practically reasonable cases. For larger angular deflections, there would be a high risk of cable slacking leading to more complex motion not covered by (1).

Remark 2 It is easy to see that also a simpler structure controller

$$
\ddot{y}=K x_{1} x_{2}
$$

is stabilizable. The term $x_{1}^{2}+\frac{1}{\Omega^{2}} x_{2}^{2}$ is included to the denominator of (34) to scale the amplitude analogously to the time-delay controllers above. This allows achieving exponential stability, which is studied in the sequel.

Lemma 4 The equivalent damping for system (1) with controller (34) is given by

$$
\zeta=\frac{K}{8 l \Omega}
$$

PROOF. Consider the linear model (9) valid under small angle assumption. Applying the feedback rule (34), the closed loop system is given by

$$
\ddot{\varphi}+\Omega^{2} \varphi+\frac{1}{l} K \frac{\varphi \dot{\varphi}}{\varphi^{2}+\frac{1}{\Omega^{2}} \dot{\varphi}^{2}} \varphi=0 .
$$

Under the oscillator response approximation (17) implying

$$
\dot{\varphi} \cong-\varphi_{0} \Omega \sin (\Omega t)
$$

the equation (40) can be simplified to

$$
\ddot{\varphi}+\Omega^{2} \varphi+\frac{K}{l \varphi_{0}^{2}} \dot{\varphi} \varphi^{2}=0 .
$$

Then, multiplying (42) by $\dot{\varphi}$ and considering (10)-(11) the equation (42) is turned to

$$
\frac{\mathrm{d}}{\mathrm{d} t}\left(\frac{1}{2} \dot{\varphi}^{2}\right)+\Omega^{2} \frac{\mathrm{d}}{\mathrm{d} t}\left(\frac{1}{2} \varphi^{2}\right)+\frac{K}{l \varphi_{0}^{2}} \dot{\varphi}^{2} \varphi^{2}=0 .
$$


Integrating (43) over the time period $t \in[0, T]$, we obtain

$$
\frac{1}{2} \dot{\varphi}^{2}(T)-\frac{1}{2} \dot{\varphi}^{2}(0)+\frac{1}{2} \Omega^{2} \varphi^{2}(T)-\frac{1}{2} \Omega^{2} \varphi^{2}(0)+\frac{K}{l \varphi_{0}^{2}} \int_{0}^{T} \dot{\varphi}^{2} \varphi^{2} \mathrm{~d} t=0,
$$

leading to

$$
\frac{-K}{l \varphi_{0}^{2}} \int_{0}^{T} \dot{\varphi}^{2} \varphi^{2} \mathrm{~d} t=\frac{1}{2} \dot{\varphi}^{2}(T)+\frac{1}{2} \Omega^{2} \varphi^{2}(T)-\frac{1}{2} \dot{\varphi}^{2}(0)-\frac{1}{2} \Omega^{2} \varphi^{2}(0) .
$$

Considering (15), the dissipated energy over the oscillation period is given by

$$
\Delta E=\frac{-K}{l \varphi_{0}^{2}} \int_{0}^{T} \dot{\varphi}^{2} \varphi^{2} \mathrm{~d} t
$$

Applying assumptions (17) and (41) on (46), we get

$$
\begin{aligned}
\Delta E=\frac{-K}{l \varphi_{0}^{2}} \Omega^{2} \varphi_{0}^{4} \int_{0}^{T} \cos (\Omega t)^{2} & \sin (\Omega t)^{2} \mathrm{~d} t= \\
& \frac{-K}{l} \Omega^{2} \varphi_{0}^{2}\left(\frac{T}{8}-\frac{\sin (4 \Omega T)}{32 \Omega}\right)=\frac{-K}{l} \Omega \varphi_{0}^{2} \frac{\pi}{4} .
\end{aligned}
$$

Considering again $\varphi(0)=\varphi_{0}, \dot{\varphi}(0)=0$ and $\ddot{\varphi}(0)=0$, implying (23), we obtain

$$
\frac{\Delta E}{E(0)}=-\pi \frac{K}{2 l \Omega}
$$

Comparing (48) and (22), the expression (39) is derived, which concludes the proof.

Note that for arbitrary chosen $\zeta \in(0,1)$ the parameter $K$ is always positive

$$
K=8 l \Omega \zeta,
$$

satisfying the constraint in Lemma 3.

Remark 3 Let us note that for both the classes of control feedback (29) (or (32)) and (34), considering the small angle assumption, the exponential stability is achieved. This is implied by the proofs of Lemma 2 and Lemma 4 . The exponential stability is implied by the existence of equivalent damping independent of $\varphi_{0} \in\left[-\frac{\pi}{2}, \frac{\pi}{2}\right]$. Note that analogous result has not been achieved so far for the alternative option when the base is fixed and the damping is achieved by varying the cable length [27]. 


\section{Case study analysis and validation}

\subsection{Sway damping of an isolated pendulum}

In the first step, a simulation based validation of the above derived controllers is performed on the isolated pendulum model (1) according to Fig. 1 - Right, assuming $\ddot{y}$ is the control input. From the algorithms based on harmonic motion of the pivot, the delay free algorithm (32) is considered. Its simulation is easy to perform as the initial conditions are point-wise, whereas they are time distributed for the time delay rule (29). Taking into account (18), the harmonic control rule is given by:

$$
\ddot{y}(t)=-4 \Delta y \Omega^{2} \frac{2 \varphi(t)\left(\zeta \varphi(t)+\frac{1}{\Omega} \dot{\varphi}(t)\right)}{\varphi^{2}(t)+\left(\zeta \varphi(t)+\frac{1}{\Omega} \dot{\varphi}(t)\right)^{2}} .
$$

Simulation based validations of both the harmonic rule (50) and the Lyapunov control rule (34) (performed in Matlab-Simulink, ode15s solver, relative tolerance 1e-12) considering $l=3 \mathrm{~m}\left(\Omega=1.81 \mathrm{~s}^{-1}\right)$ and $\varphi(0)=\frac{\pi}{2}$ are shown in Fig. 2 for two values of assigned equivalent damping $\zeta=0.05$ and $\zeta=0.20$. By (8) and (39), the free parameters of the controllers are given as $\Delta y=0.15 \mathrm{~m}$ and $K=2.17$ for $\zeta=0.05$ and $\Delta y=0.6 \mathrm{~m}$ and $K=8.68$ for $\zeta=0.20$, respectively. It can be seen in Fig. 2 that the desired exponential amplitude decay with predefined $\zeta$ is almost perfectly matched, despite the assumptions applied for derivation of the equivalent damping and despite violation of the small angle assumption. For $\zeta=0.05$, the responses of the two controllers are almost identical. This is not the case for $\zeta=0.20$, where a difference in oscillation period can be explicitly observed. The results in Fig. 2 also nicely confirm the exponential stability addressed in Remark 3. The exponential stability of the closed loop is achieved thanks to the limit-cycle motion of the control action. An analogous phenomenon cannot be observed in the class of linear systems.

\subsection{Master-slave mechatronic control of pivot position with experimental val- idation}

In the next stage, we consider the extended and more realistic model (1)(2) according to Fig. 1-Mid, where the mass of the pivot base is given by $m_{1}$. The objective is to design a control scheme to follow the pivot position trajectory $y$ generated by one of the nonlinear algorithms proposed above. For the successful performance, it is crucial that the set-point $y_{\mathrm{s}}$ trajectory of a frequency $2 \Omega \sqrt{1-\zeta^{2}}$ is followed with no shift. A repetitive scheme with a 

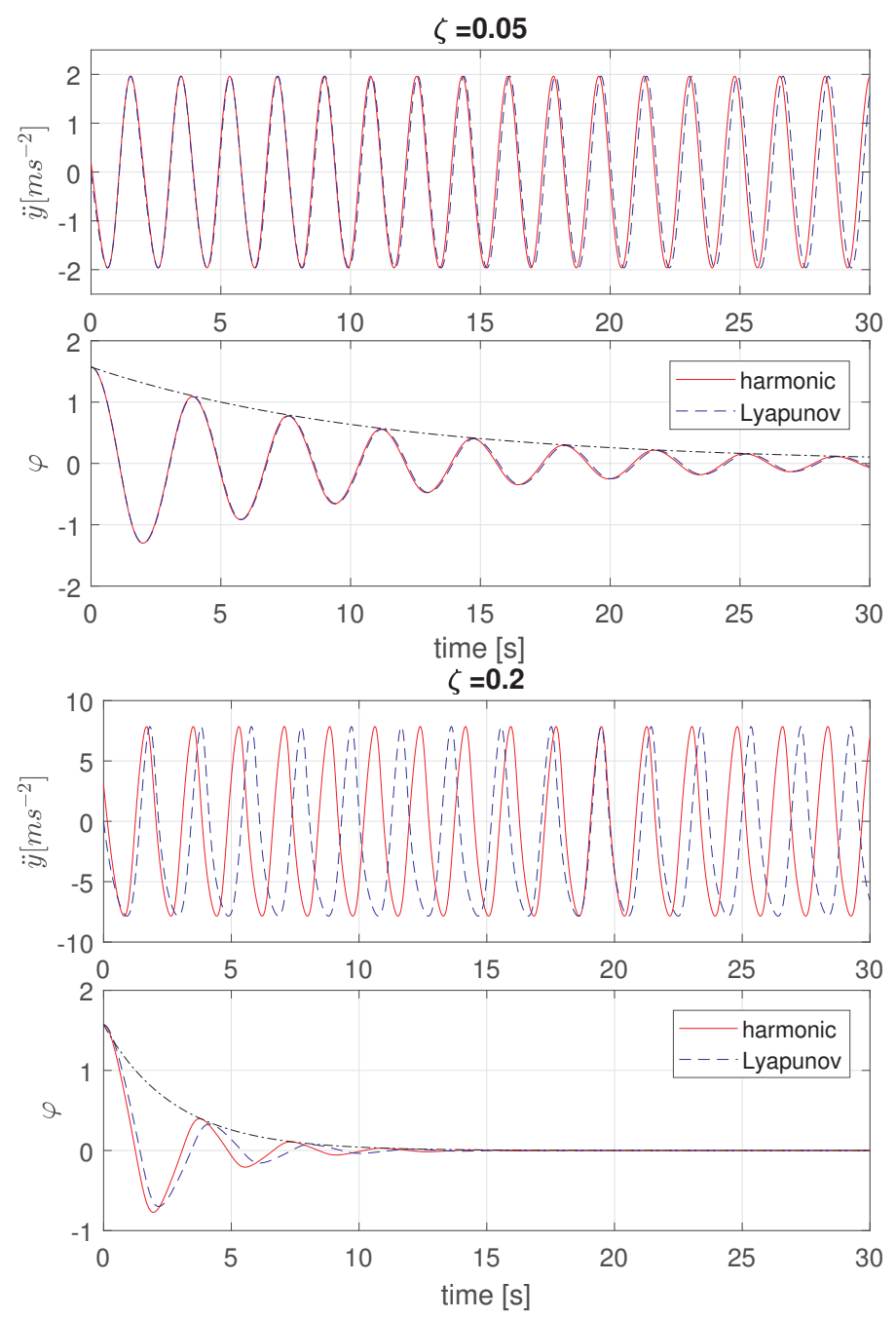

Fig. 2. Simulation results of (1) with i) the harmonic control rule (50), and ii) Lyapunov control rule (34), considering two values of assigned equivalent damping $\zeta=\{0.05,0.20\}$. The dash-dotted line $\left(\varphi_{0} e^{-\zeta \Omega t}\right)$ indicates the pre-defived exponential decay rate.

time-delay compensator is a standard tool for this task [32]. A disadvantage of the repetitive control is that a time-delay filter is included to the feedback loop implying the undesirable closed-loop neutrality. In order to avoid the difficulty to deal with control design of an infinite-order system, we take an advantage of time-delay controller (29) to compensate the time-shift between the reference and the controlled output directly by shortening the delay $\tau_{2}$.

In the first stage, we design a slave PD controller for the system (2). Linearizing the system by neglecting the effect of centrifugal and inertia forces of the bob and introducing the control force increment $\Delta F(t)=F(t)-F_{\text {nom }}$, where the nominal force is given as $F_{\text {nom }}=\left(m_{1}+m_{2}\right) g$, the model for the 
controller design is given by

$$
\left(m_{1}+m_{2}\right) \ddot{y}(t)+b \dot{y}(t)=\Delta F(t) .
$$

$\mathrm{PD}$ controller is considered in the form

$$
\Delta F=r_{\mathrm{p}}\left(y_{\mathrm{s}}-y(t)\right)-r_{\mathrm{d}} \dot{y}(t)
$$

Assigning a double root $s_{1,2}=-p$ to the characteristic equation of the closedloop system (51)-(52)

$$
\left(m_{1}+m_{2}\right) s^{2}+\left(b+r_{\mathrm{d}}\right) s+r_{\mathrm{p}}=0,
$$

the parameters of the PD controller are given by

$$
\begin{gathered}
r_{\mathrm{p}}=p^{2}\left(m_{1}+m_{2}\right), \\
r_{\mathrm{d}}=2 p\left(m_{1}+m_{2}\right)-b .
\end{gathered}
$$

For adjusting the reference signal, the time and gain shift are determined using the following parametrization

$$
\begin{gathered}
t_{\phi}=\frac{1}{2 \Omega \sqrt{1-\zeta^{2}}} \angle G\left(j 2 \Omega \sqrt{1-\zeta^{2}}\right) \\
m_{G}=\left|G\left(j 2 \Omega \sqrt{1-\zeta^{2}}\right)\right|,
\end{gathered}
$$

where

$$
G(s)=\frac{r_{\mathrm{p}}}{\left(m_{1}+m_{2}\right) s^{2}+\left(b+r_{\mathrm{d}}\right) s+r_{\mathrm{p}}} .
$$

Finally, the master controller to generate the pivot position set-point is adjusted from (29) to

$$
y_{\mathrm{s}}(t)=y_{0}+\frac{\Delta y}{m_{G}} \frac{\varphi^{2}\left(t-\left(\tau_{2}+t_{\phi}\right)\right)-e^{-\zeta \pi} \varphi^{2}\left(t-\left(\tau_{2}+t_{\phi}\right)-\tau_{1}\right)}{\varphi^{2}\left(t-\left(\tau_{2}+t_{\phi}\right)\right)+e^{-\zeta \pi} \varphi^{2}\left(t-\left(\tau_{2}+t_{\phi}\right)-\tau_{1}\right)},
$$

assuming $\tau_{2}>-t_{\phi}$. The overall control scheme is given in Fig. 3. Note that if the overall closed-loop dynamics is set up too slow, it may happen that the overall time-delay $\tau_{2}+t_{\phi}$ is negative. In this case, a faster controller setting needs to be achieved by increasing the design parameter $p$.

In order to perform validation of the above proposed master-slave control loop, the laboratory set-up shown in Fig. 4 with a mechatronic control system has been built. The movement of the cart is ensured by a servomotor connected to the cart by a toothed belt. The position of the cart $y$ as well as the rope angle $\varphi$ are measured by magnetic sensors. The rope angle is transferred to the sensor by a shaft, which is nested in low-friction bearings. The control algorithms are 


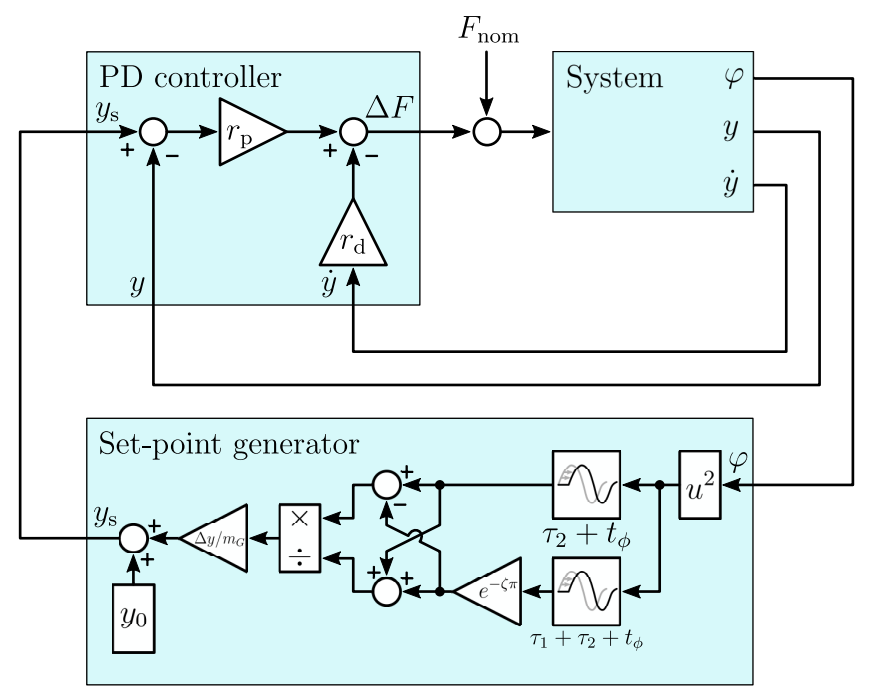

Fig. 3. Model of the overall closed loop consisting of the model (1)-(2), harmonic set-point generator - master controller (59) and the slave PD controller (52).
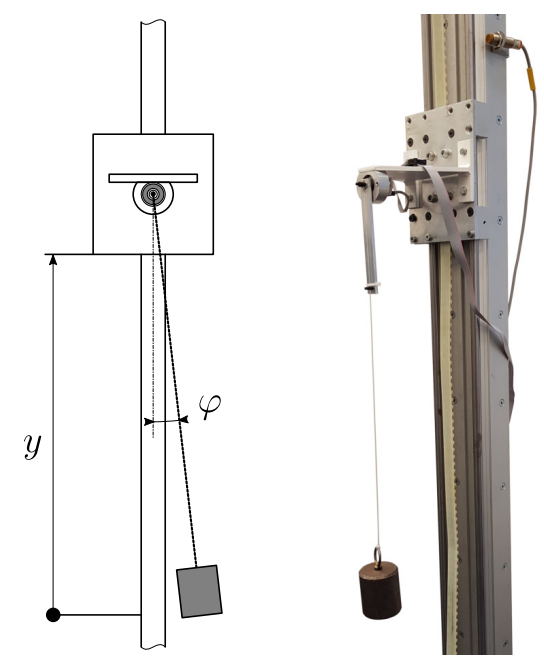

Fig. 4. Experimental set-up

implemented in the NI CompactRIO control unit combining a fast real-time loop with FPGA, where the sensor information processing is performed. A differential line transceiver/receiver is used for both magnetic sensors signal conditioning. A magnetic strip attached next to cart rails with pole pitch $0.04 \mathrm{~mm}$ is used for precise position measurement. The velocity of the cart $\dot{y}$ necessary for PD controller algorithm is evaluated using difference method in high-speed loop implemented in FPGA with support of the second-order low-pass filter ensuring a satisfactory noise attenuation. The servo torque and the control force $\Delta F$ are controlled by bipolar analog voltage signal generated by the control unit.

The following parameters of the set-up have been measured and identified: $l=0.9 \mathrm{~m}, m_{1}=1.52 \mathrm{~kg}, m_{2}=0.85 \mathrm{~kg}, b=3.4 \mathrm{kgs}^{-1}$. The design parameter 

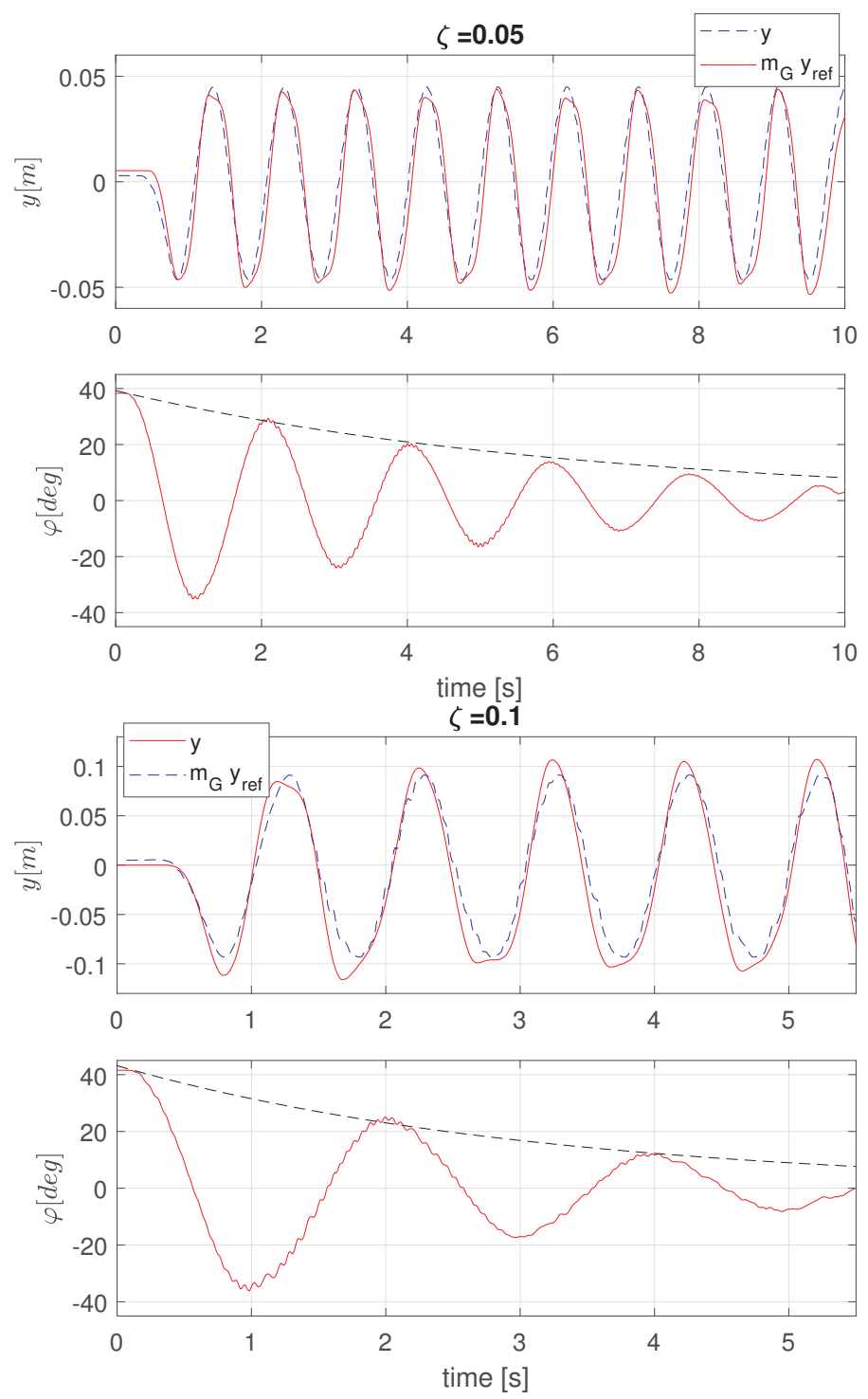

Fig. 5. Results of experiment on the set-up in Fig. 4 with the PD controller (52) and set-point generator (59) for $\zeta=\{0.05,0.10\}$. The dashed line $\left(\varphi_{0} e^{-\zeta \Omega t}\right)$ indicates the pre-defived exponential decay rate.

of the controller was assigned to $p=25 \mathrm{~s}^{-1}$, providing the parameters $r_{\mathrm{p}}=$ $1481 \mathrm{Nm}^{-1}$ and $r_{\mathrm{d}}=115 \mathrm{kgs}^{-1}, t_{\phi}=-0.078 \mathrm{~s}$ and $m_{G}=0.93$. Both the PD controller and set-point generator (59) were implemented in NI LabVIEW in discrete form with time sampling $\Delta t=0.01 \mathrm{~s}$.

The validation was performed for $\zeta=0.05(\Delta y=0.045 \mathrm{~m})$ and $\zeta=0.10$ $(\Delta y=0.090 \mathrm{~m})$. As it can be seen in Fig. 5 , the prescribed equivalent damping has been achieved for both cases ${ }^{1}$. It is worth mentioning that the cart motion 1

Video record from the experiments can be seen at https://control.fs.cvut.cz/en/aclab/updowndamp 
is stopped as soon as the amplitude falls below the predefined $\Delta y_{\min }$, which needs to be larger than the measurement and system noise. Otherwise, the control system will induce chaotic motion of the cart-pendulum system.

\subsection{Quadcopter motion control}

Finally, the simulation based validation is performed for the quadcopter set-up according to Fig. 1 - Left. Dynamics of the quadcopter carrying the suspended load is given by the following set of nonlinear equations

$$
\mathbf{M}(\mathbf{x}(t)) \ddot{\mathbf{x}}(t)+\mathbf{C}(\mathbf{x}(t)) \dot{\mathbf{x}}(t)+\mathbf{Q}(\mathbf{x}(t))=\mathbf{L}(\mathbf{x}(t)) \mathbf{u}(t),
$$

where $\mathbf{x}(t)=[x, y, \theta, \varphi]^{\mathrm{T}}$ with $x, y$ denoting position coordinates of the quadcopter's center of the mass, $\theta$ is the pitch angle of the quadcopter and $\varphi$ is the angle between vertical axis $y$ and the cable as indicated in Fig. 6. Vector $\mathbf{u}(t)=[T, M]^{\mathrm{T}}$ is the vector of controls - the thrust $T$ and torque $M$ generated by the motors with the propellers. The state dependent matrices of the motion and the system parameters are given in the Appendix A.
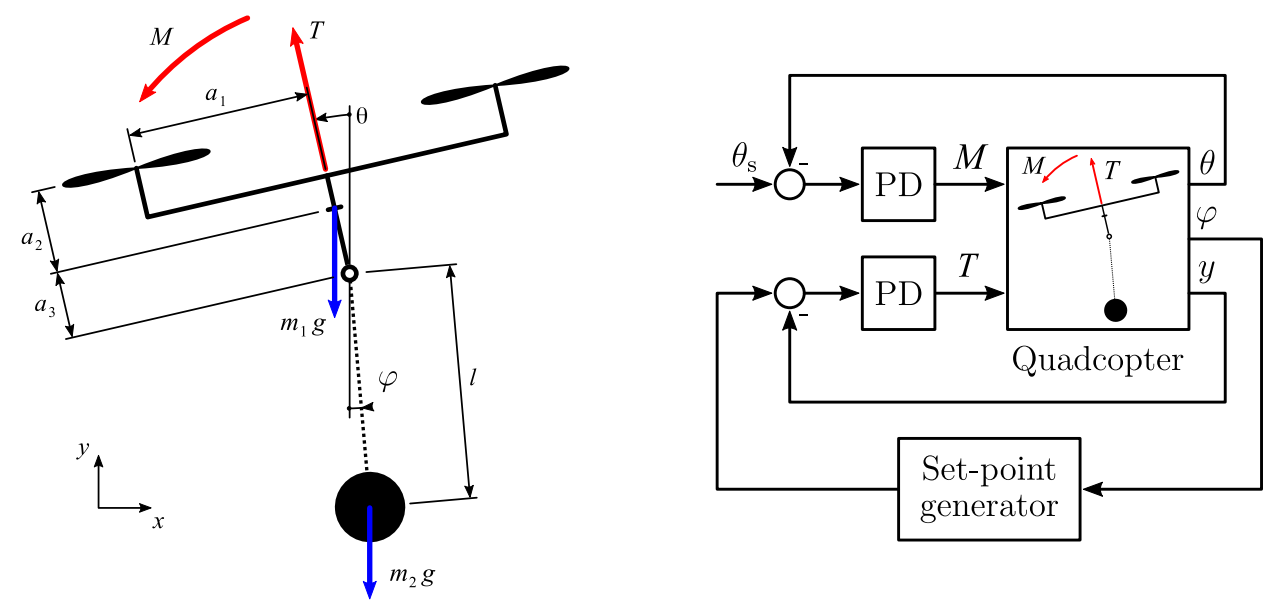

Fig. 6. Quadcopter with suspended load geometry (left) and control scheme (right).

The quadcopter control system consists of two PD feedback loops shown in Fig. 6 - right. The first loop controls the pitch angle $\theta$ by the moment $M$, while the other controls the motion in $y$ axis via the thrust $T$. The set-point for the pitch angle is set to zero $\theta_{\mathrm{s}}=0$ while the set-point $y_{\mathrm{s}}$ is determined by the aforementioned control rule (52)-(59) shown in Fig. 3 with controller parameters $r_{\mathrm{p}, y}=30, r_{\mathrm{d}, y}=11, m_{G}=0.6565, t_{\phi}=-0.3463 \mathrm{~s}$ determined by (54), (55), (56) and (57) for the model (60) linearized in the equilibrium point.

Simulation results (Matlab-Simulink, ode15s, rel. tol. 1e-12) for two values of prescribed equivalent damping $\zeta=\{0.05,0.20\}$ can be seen in Fig. 7 for 

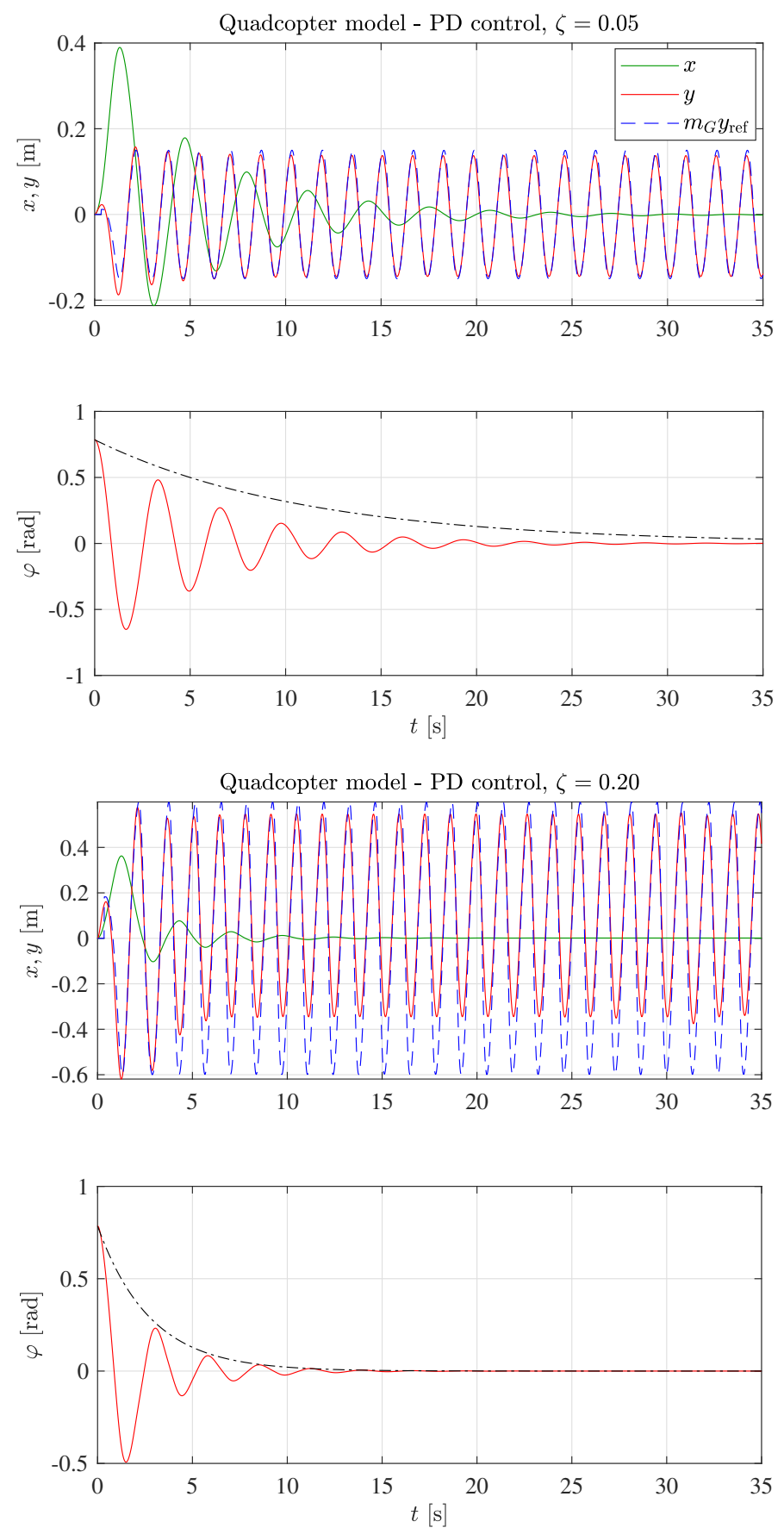

Fig. 7. Simulation results of the quadcopter model (60) controlled by PD controller (52) and the delay based rule (59) for generating the setpoint, according to Fig. 3 considering two values of prescribed equivalent damping $\zeta=\{0.05,0.20\}$. The dashdotted line $\left(\varphi_{0} e^{-\zeta \Omega t}\right)$ indicates the pre-defived exponential decay rate.

$\varphi_{0}=\frac{\pi}{4}$. In both cases, the oscillations are well damped. For the predefined $\zeta=0.05$, larger than prescribed damping is achieved. It is due to the fact that the coupling of the quadcopter and the undamped pendulum already provides damped response with $\zeta=0.04$. For $\zeta=0.20$, a very good match of 


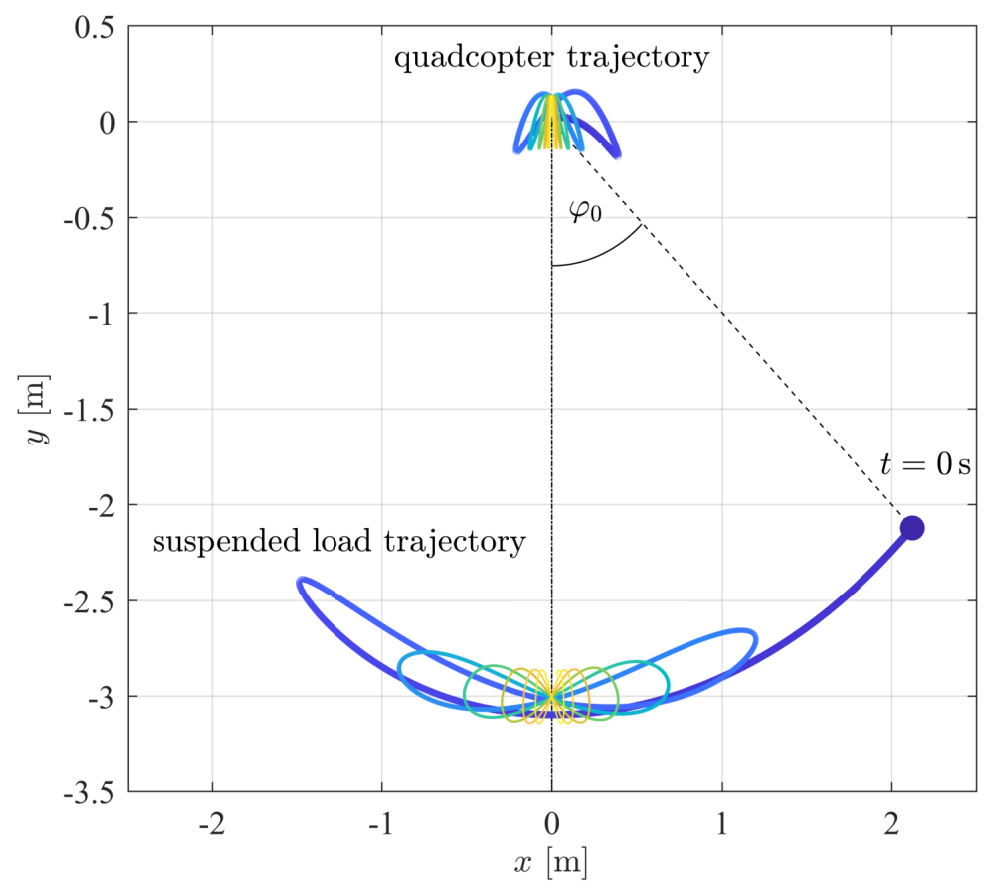

Fig. 8. Trajectory of the centres of gravity of the isolated quadcopter and the suspended load for $\zeta=0.05$

the predefined decay is achieved, even though the $y$ position does not follow the generated set-point exactly. As it can be seen in Fig. 7 and also in Fig. 8 with vizualization of the 2-D motion of the centres of gravity of the isolated quadcopter and the pendulum, the whole system works also under motion in the $x$-axis at the beginning of the responses imposed by the internal couplings between these two bodies. Achieving very good results under these aspects (not considered in the design) shows a certain level of robustness of the proposed scheme. However, such robustness issues are out of the scope of this paper and they have not been addressed.

\section{Conclusion}

In this paper, design and analysis of nonlinear controllers for sway damping of suspended payload by moving the pivot base in vertical direction have been performed. The first proposed algorithm (with two different feedback implementations) is based on utilizing the momentum effect of the inertia force acting on the payload under the periodic motion. The other algorithm is designed based on Lyapunov's second method. Unlike in the analogous algorithms for sway damping by adjusting the cable length, a solution with exponential amplitude damping is achieved. The derivation of equivalent damping with the ideal second order oscillator was performed by balancing the dissipation en- 
ergy over the one oscillation period. The derived algorithms are validated in a thorough case study section. This includes the experimental validation as well as simulation based validation on a 2-D nonlinear model of a quadcopter with suspended payload, which was in fact the motivation to study the given problem. In future, some experimental validation on a physical quadcopter is to be performed. In the crane applications, the proposed method could be used to damp the residual vibrations when the desired crane position is reached. Another application can be found in mechatronic systems and flexible robotics where analogous problems uncontrollable by linear methods can occur.

\section{A Appendix - State dependent matrices and parameters of the quadcopter model}

The state dependent matrices of the nonlinear model (60) are given as

$$
\begin{aligned}
& \mathbf{M}(\mathbf{x}(t))= \\
& {\left[\begin{array}{cccc}
m_{1}+m_{2} & 0 & a_{3} m_{2} \cos (\theta) & l m_{2} \cos (\varphi) \\
0 & m_{1}+m_{2} & a_{3} m_{2} \sin (\theta) & l m_{2} \sin (\varphi) \\
a_{3} m_{2} \cos (\theta) & a_{3} m_{2} \sin (\theta) & m_{2} a_{3}^{2}+I_{1} & a_{3} l m_{2} \cos (\varphi-\theta) \\
l m_{2} \cos (\varphi) & l m_{2} \sin (\varphi) & a_{3} l m_{2} \cos (\varphi-\theta) & m_{2} l^{2}+I_{2}
\end{array}\right]} \\
& \mathbf{C}(\mathbf{x}(t))=\left[\begin{array}{cccc}
c_{x} & 0 & 0 & 0 \\
0 & c_{y} & 0 & 0 \\
0 & 0 & c_{\theta} & 0 \\
0 & 0 & 0 & 0
\end{array}\right] \\
& \mathbf{Q}(\mathbf{x}(t))=\left[\begin{array}{c}
-l m_{2} \sin (\varphi) \dot{\varphi}^{2}-a_{3} m_{2} \sin (\theta) \dot{\theta}^{2} \\
l m_{2} \cos (\varphi) \dot{\varphi}^{2}+a_{3} m_{2} \cos (\theta) \dot{\theta}^{2}+\left(m_{1}+m_{2}\right) g \\
-a_{3} l m_{2} \sin (\varphi-\theta) \dot{\varphi}^{2}+a_{3} m_{2} g \sin (\theta) \\
a_{3} l m_{2} \sin (\varphi-\theta) \dot{\theta}^{2}+l m_{2} g \sin (\varphi)
\end{array}\right],
\end{aligned}
$$

where $g=9.81 \mathrm{~ms}^{-2}, m_{1}=1 \mathrm{~kg}, I_{1}=0.01 \mathrm{kgm}^{2}, a_{1}=0.20 \mathrm{~m}, a_{2}=0.02 \mathrm{~m}$, $a_{3}=0.03 \mathrm{~m}, m_{2}=0.2 \mathrm{~kg}, I_{2}=0 \mathrm{kgm}^{2}, l=3 \mathrm{~m}, c_{x}=1 \mathrm{kgs}^{-1}, c_{y}=1 \mathrm{kgs}^{-1}$, $c_{\theta}=1 \mathrm{kgs}^{-1}$. 


\section{Acknowledgements}

The presented research has been supported by the Ministry of education of Czech Republic under the INTER-EXCELLENCE project INTER-ACTION LTAUSA17103.

\section{References}

[1] E. M. Abdel-Rahman, A. H. Nayfeh, Z. N. Masoud, Dynamics and control of cranes: A review, Journal of Vibration and Control 9 (7) (2003) 863-908.

[2] W. Singhose, D. Kim, M. Kenison, Input shaping control of double-pendulum bridge crane oscillations, Journal of Dynamic Systems, Measurement, and Control 130 (3) (2008) 034504.

[3] J. Vaughan, D. Kim, W. Singhose, Control of tower cranes with doublependulum payload dynamics, IEEE Transactions on Control Systems Technology 18 (6) (2010) 1345-1358.

[4] W. Singhose, Command shaping for flexible systems: A review of the first 50 years, International Journal of Precision Engineering and Manufacturing 10 (4) (2009) 153-168.

[5] J. J. Potter, C. J. Adams, W. Singhose, A planar experimental remotecontrolled helicopter with a suspended load, IEEE/ASME Transactions on Mechatronics 20 (5) (2015) 2496-2503.

[6] K. L. Sorensen, W. Singhose, S. Dickerson, A controller enabling precise positioning and sway reduction in bridge and gantry cranes, Control Engineering Practice 15 (7) (2007) 825-837.

[7] P. Beneš, M. Valášek, Z. Šika, J. Zavřel, J. Pelikán, Shavo control: The combination of the adjusted command shaping and feedback control for vibration suppression, Acta Mechanica 230 (5) (2019) 1891-1905.

[8] T. Vyhlídal, M. Hromčík, V. Kučera, M. Anderle, On feedback architectures with zero-vibration signal shapers, IEEE Transactions on Automatic Control 61 (8) (2016) 2049-2064.

[9] C.-Y. Chang, H. W. Lie, Real-time visual tracking and measurement to control fast dynamics of overhead cranes, IEEE Transactions on Industrial Electronics 59 (3) (2012) 1640-1649.

[10] D. Chwa, Nonlinear tracking control of 3-D overhead cranes against the initial swing angle and the variation of payload weight, IEEE Transactions on Control Systems Technology 17 (4) (2009) 876-883. 
[11] H. Chen, Y. Fang, N. Sun, A swing constraint guaranteed MPC algorithm for underactuated overhead cranes, IEEE/ASME TRANSACTIONS ON MECHATRONICS 21 (5) (2016) 2543-2555.

[12] S. Yang, B. Xian, Robust control design for the quadrotor UAV with a suspended payload, in: 2018 IEEE 8th Annual International Conference on CYBER Technology in Automation, Control, and Intelligent Systems (CYBER), IEEE, 2018, pp. 469-473.

[13] G. Bartolini, A. Pisano, E. Usai, Second-order sliding-mode control of container cranes, Automatica 38 (10) (2002) 1783-1790.

[14] T. A. Le, G.-H. Kim, M. Y. Kim, S.-G. Lee, Partial feedback linearization control of overhead cranes with varying cable lengths, International Journal of Precision Engineering and Manufacturing 13 (4) (2012) 501-507.

[15] C. Vázquez, J. Collado, L. Fridman, Control of a parametrically excited crane: A vector Lyapunov approach, IEEE Transactions on Control Systems Technology 21 (6) (2013) 2332-2340.

[16] N. Sun, Y. Fang, Nonlinear tracking control of underactuated cranes with load transferring and lowering: Theory and experimentation, Automatica 50 (9) (2014) 2350-2357.

[17] P. J. Cruz, M. Oishi, R. Fierro, Lift of a cable-suspended load by a quadrotor: A hybrid system approach, in: 2015 American Control Conference (ACC), IEEE, 2015, pp. 1887-1892.

[18] B. Piccoli, J. Kulkarni, Pumping a swing by standing and squatting: Do children pump time optimally?, IEEE Control Systems 25 (4) (2005) 48-56.

[19] V. Sevrez, E. Berton, G. Rao, R. J. Bootsma, Regulation of pendulum length as a control mechanism in performing the backward giant circle in gymnastics, Human Movement Science 28 (2) (2009) 250-262.

[20] M. Pinsky, A. Zevin, Oscillations of a pendulum with a periodically varying length and a model of swing, International journal of non-linear mechanics 34 (1) (1999) 105-109.

[21] L. Akulenko, S. Nesterov, The stability of the equilibrium of a pendulum of variable length, Journal of applied mathematics and mechanics 73 (6) (2009) 642-647.

[22] D. S. Stilling, W. Szyszkowski, Controlling angular oscillations through mass reconfiguration: A variable length pendulum case, International Journal of NonLinear Mechanics 37 (1) (2002) 89-99.

[23] S. Okanouchi, K. Yoshida, I. Matsumoto, H. Kawabe, Damping angular oscillations of a pendulum under state constraints, IFAC Proceedings Volumes 41 (2) (2008) 7735-7742.

[24] E. Gandino, S. Marchesiello, A. Bellino, A. Fasana, L. Garibaldi, Damping effects induced by a mass moving along a pendulum, Shock and Vibration 2014. 
[25] T. Vyhlídal, M. Anderle, J. Bušek, S.-I. Niculescu, Time-delay algorithms for damping oscillations of suspended payload by adjusting the cable length, IEEE/ASME Transactions on Mechatronics 22 (5) (2017) 2319-2329.

[26] K. Yoshida, I. Kawanishi, H. Kawabe, Stabilizing control for a single pendulum by moving the center of gravity: theory and experiment, in: Proceedings of the 1997 American Control Conference (Cat. No. 97CH36041), Vol. 5, IEEE, 1997, pp. 3405-3410.

[27] M. Anderle, W. Michiels, S. Čelikovský, T. Vyhlídal, Damping a pendulum's swing by string length adjustment-design and comparison of various control methods, in: 2019 American Control Conference (ACC), IEEE, 2019, pp. 43994405 .

[28] J. Bušek, M. Kuře, M. Hromčík, T. Vyhlídal, Control design with inverse feedback shaper for quadcopter with suspended load, in: ASME 2018 Dynamic Systems and Control Conference, American Society of Mechanical Engineers, 2018, pp. V003T40A009-V003T40A009.

[29] M. Kuře, J. Bušek, T. Vyhlídal, S.-I. Niculescu, Damping oscillation of suspended payload by up and down motion of the pivot base - time delay algorithms for UAV applications, IFAC-PapersOnLine 52 (18) (2019) 121-126.

[30] G. L. Baker, J. A. Blackburn, The pendulum: A case study in physics, Oxford University Press, 2005.

[31] J. LaSalle, S. Lefschetz, Stability by Lyapunovs second method with applications, Academic Press, New York, NY, 1961.

[32] Y. Wang, F. Gao, F. J. Doyle III, Survey on iterative learning control, repetitive control, and run-to-run control, Journal of Process Control 19 (10) (2009) 15891600 . 